\title{
The Role of Knowledge Sharing in Organizational Performance
}

\author{
Zainab Al Kashari \\ Faculty of Engineering and Information Technology, The British University in Dubai \\ Dubai, United Arab Emirates \\ Fatma Al Taheri \\ Faculty of Engineering and Information Technology, The British University in Dubai \\ Dubai, United Arab Emirates
}

\begin{abstract}
With the tremendous development in the current world, it has become necessary for organizations to convey with the rhythm of development in order to achieve the advantage of constructive competition with other organizations in their field. Where knowledge management is considered one of the methods used to raise the level of development, increase revenue and competitive advantage. The purpose of this paper is to discover the role of knowledge sharing in the organization in terms of increasing the effectiveness and efficiency, the significance of knowledge sharing, relationship between knowledge sharing and organizational leadership, the issues of knowledge sharing and how can be addressed. The applied method in this study is Qualitative Research which includes the use of non-statistical data to explain or explore a certain phenomenon. This paper has been failed to include the correspondence between knowledge sharing, employee motivation and performance, as this topic is also reflective of the extended role of knowledge sharing in the efficiency, satisfaction and retention of employees.
\end{abstract}

Keywords: Knowledge Sharing; Knowledge Management; Knowledge and Leadership; Knowledge Issues; The Role of Knowledge; Learning Organization;

DOI: $10.7176 / \mathrm{IKM} / 10-2-05$

Publication date: February $29^{\text {th }} 2020$

\section{Introduction}

In a constantly transforming and development market and industry environment, it has become increasingly important for organizations to keep in pace in order to achieve sustainable competitive advantage. One of the widely recognized approaches that organizations implement is knowledge management, as they put emphasis on the significance of knowledge in their business process. Knowledge management (KM), as indicated by Gonzalez and Martins (2014), is an integral component of organizational development which includes organizational culture and structure. It focuses on effective handling, management, storage and transfer of knowledge being the primary resource of organizations specifically in the 21 st century.

Alongside the growing significance of KM in organizations, it is consequently identified that distribution, accessibility and usability of knowledge should be one of its main components. Such process is commonly termed as knowledge sharing (KS) which is also used interchangeably with knowledge transfer (KT). There has always been vagueness as to the differences between the two; nonetheless, knowledge sharing, according to Paulin and Suneson (2012, p. 83), is defined as "the exchange of knowledge between and among individuals, and within and among teams, organizational units, and organizations. This exchange may be focused or unfocused, but it usually does not have a clear priori objective." Knowledge sharing, being the third step in the knowledge management process, entails how knowledge itself can be adapted and evolved, thereby allowing people within the organization to access and use it to further their learning (Kumar \& Kalva, 2015; Alqaryouti, Alqudah \& Shaalan, 2016).

The purpose of this paper is to understand the context of knowledge sharing, that is, by exploring all the associated relevant concepts including the issues and problems surrounding it as well as the solutions that organizations implement to address said challenges. In addition, this study aims to review the role of knowledge sharing in furthering the KM process and in propelling organizational efficiency. With an emphasis on knowledge sharing and its role in the organization, this report will expand learning on this matter.

In this aspect, this study focuses on the following research questions about knowledge sharing that would be answered through the use of existing resources which include journal articles and reliable websites:

1. How significant is knowledge sharing in fostering efficiency in knowledge management?

2. How does knowledge sharing impact the organization?

3. What is the relationship between knowledge sharing and organizational leadership?

4. What are the issues about knowledge sharing? What are their respective solutions? 


\section{Literature Review}

The birth of knowledge management is rooted on the significance of knowledge in the organization's pursuit of sustainable competitive advantage, given the dynamic environment it moves in. Knowledge management, as a scientific discipline, has then become an increasingly interesting topic both in research and in practice (Fteimi \& Lehner, 2016). One of the most essential parts in KM is knowledge sharing which is crucial to develop in the organization, particularly between and among teams. In return, employees are given the opportunity to provide, share and apply knowledge that would yield better outcomes for the organization. However, Zheng (2017) underlined that "knowledge sharing is not communication" (p. 52). This means that it is impossible to freely distribute knowledge similar to how goods are handled and despite not being communication, knowledge sharing is built on the relationship between parties - one having knowledge and the other obtaining knowledge. It is additionally revealed that knowledge sharing has four major characteristics such as: (1) KS as an individual behaviour; (2) KS is "a voluntary, proactive, behavioural awareness" (p. 52); (3) KS is governed by several standards and regulations relating to ethics and code of conduct; and (4) the results of KS are comprised of two or more parties.

There are various accounts entailing that knowledge sharing is an action between two individuals wherein one party has the knowledge and shares it while the other only collects the knowledge. Nonetheless, there are also researchers, as mentioned in the study by Pangil and Nasurddin (2013), who indicated that knowledge sharing is a mutual exchange between parties, which means that both parties share and collect knowledge from each other. This mechanism denotes a collaborative learning between two parties. In relation to this, knowledge sharing can be different for every type of knowledge. For one, sharing of tacit knowledge is deemed more challenging than that of explicit knowledge. This is due to the fact that the former centers on individual experience which consequently includes personal attitudes and standpoints. People usually have a hard time expressing this type of knowledge to others as it is also shared in other methods of expression instead of the formal language use. On the other hand, explicit knowledge can be shared through books, databases, video clips and the like. It happens very easily and people do not have to be encouraged to share. Commonly, explicit knowledge sharing enables employees to be more efficient with their work, thereby exhibiting productivity and competence.

According to Lee (2018), the primary activity in the knowledge management process is knowledge sharing, which explains the reason why organizations are motivated to put significant regard on this aspect as they acknowledge its positive implications and benefits. Knowledge sharing is found to be positively linked with cost reduction, enhanced efficiency, improved performance of both the employees and the entire organization and fostered teamwork within the organization. As employees share knowledge, they can exploit and capitalize knowledge-based resources that would be helpful in creating innovation and in improving business process, thus leading to increased organizational competitiveness. This denotes that knowledge sharing is more than just exchange of information; but, it is actually a tool that promotes creativity and innovativeness among employees. Individual creativity is encouraged through the use of knowledge resources and as the people work together, such collaboration can help generate better ideas for improvement of the organization.

Considering the potential benefits that knowledge sharing brings to the individual employees and to the organization, the need for the activity to be effective is underpinned. This means that transfer of the knowledge between parties must be effective from the very beginning, which is, starting "from the individual to the team, from the team to the knowledge management process level, from the knowledge management process to the organization, and from the organization to the industry" (Turner, Zimmerman \& Allen, 2012, p. 21). From this perspective, it can be further noted that knowledge sharing is a never-ending process. As knowledge increases and evolves the process of knowledge sharing continues. According to Rusuli and Tasmin (2010), this is explained by how personal knowledge of an individual employee is transformed into organizational knowledge, making this resource valuable to the organization. The continuous transformation of knowledge is termed as knowledge conversion and has four modes as per the SECI process: [1] Socialization, which is aimed at tacit knowledge sharing among individuals; [2] Externalization, which is the articulation of tacit knowledge into explicit contexts; [3] Combination, which refers to the integration of various components of explicit knowledge; and [4] Internalization, which is the embodiment of explicit knowledge into tacit knowledge (see figure below). 


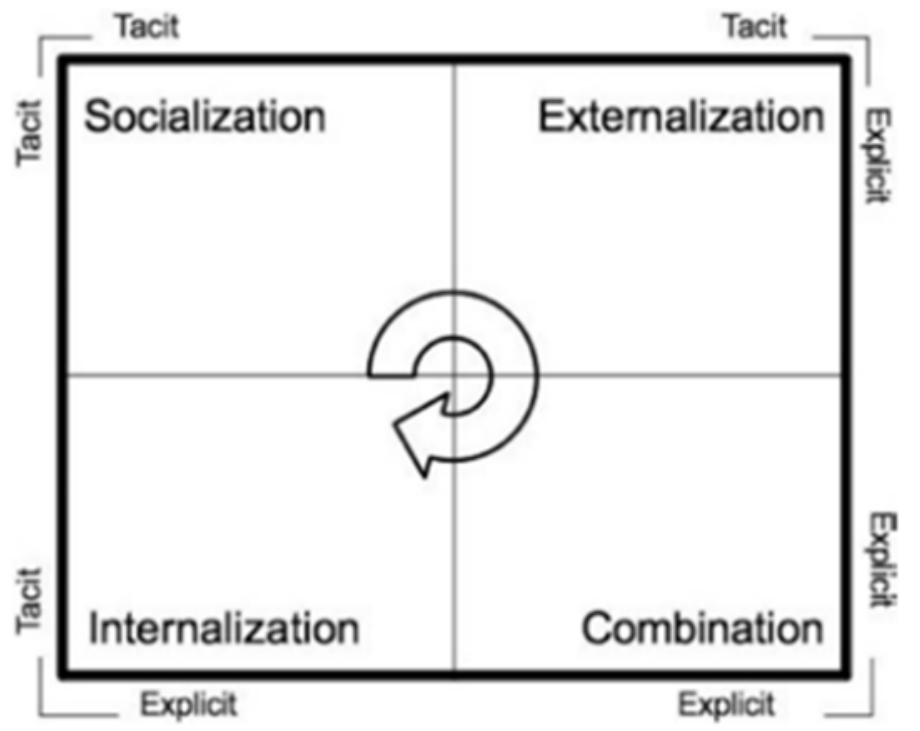

Figure 1: SECI Process Model

Source: Rusuli \& Tasmin (2010)

\section{Methodology}

One of the research designs utilized in many studies is the Qualitative Research which involves the use of nonstatistical data to explain or explore a certain phenomenon. Unlike Quantitative Research, it emphasizes on using information instead of numbers (Shakouri, 2014). In this study, this particular method is applied; however, researchers did not conduct a survey or an interview with a number of participants. Instead, secondary research is utilized through the use of existing sources such as journal articles and credible websites. With secondary data analysis, the researchers no longer need to dedicate time and effort to conduct data collection procedures. At the same time, this method allows a more in-depth exploration of the topic, given the different standpoints in the studies used. It helps expand and reinterpret the data gathered, making the discussion more investigative in nature (Greenhoot \& Dowsett, 2012).

The very core of this study is reflective on the choice of sources used. To mention, the researchers take caution in selecting the studies used as basis of the arguments presented in this paper, strictly limiting on journal articles. Most of the sources were obtained online as there are several free online journal articles that can be accessed. However, there are also other sources that require subscription before they can be accessed and this is one of the primary challenges of the researchers. Needless to say, the sources found are relevant to the topic and these have helped largely in the completion of this research. Furthermore, the data collection process is estimated to have lasted for two weeks. Keywords used include knowledge management, knowledge sharing and knowledge transfer. Approximately, 20 journal articles are used in this study - all of which are associated with knowledge sharing and knowledge management in general.

\section{Results}

\subsection{How significant is knowledge sharing in fostering efficiency in knowledge management?}

In understanding the role of knowledge sharing in the knowledge management process, it is also important to underpin that knowledge management is an essential factor in organizational efficiency and success. According to Intezari, Taskin and Pauleen (2017), knowledge management process includes a variety of activities and this includes knowledge sharing; however, it is consequently underscored that these activities are required to be efficient and systematic. This then entails that the effectiveness of knowledge sharing contributes largely to the overall effectiveness of knowledge management in the organization, alongside other elements of KM infrastructure as shown on the figure below. It is also an avenue for employee learning and socialization wherein new ideas are generated by the employees to further organizational innovations. 


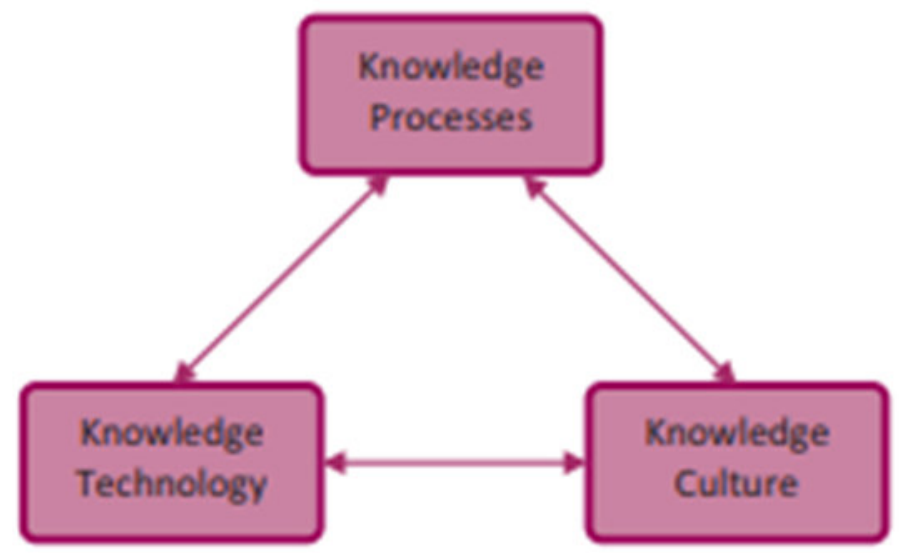

Figure 2: Elements of KM infrastructure and the relationships of each element

Source: Intezari, Taskin \& Pauleen, 2017

As indicated by Mohajan, Islam and Shome (2017), knowledge sharing has a positive implication in knowledge management environment and efficiency, denoting that individual knowledge is not immediately transformed to organizational knowledge without knowledge sharing. Nonetheless, the employees and their behaviour toward this particular process are highly significant in the effectiveness of knowledge conversion. As people share knowledge to develop ideas for the organization, it is therefore emphasized that knowledge sharing largely affects the culture of the organization, the creation of learning as well as organizational knowledge and promotion of organizational innovation. This indicates the contention that there is a positive relationship between efficiency in knowledge sharing, efficiency of the KM process and organizational efficiency.

\subsection{How does knowledge sharing impact the organization?}

Knowledge sharing, apart from having a positive impact on the KM process, has significant value in the organization as repeatedly highlighted in the previous pages of this study. As a matter of fact, creating a knowledge sharing culture is what most organizations are working hard for because of the benefits it yields. Intranets are commonly used by employees to share, exchange and access knowledge; however, implementing such system is not without its challenges considering that there are employees who resist to knowledge sharing. In that aspect, organizations are prompted to further their methods to improve the knowledge sharing capabilities and behaviours of the employees. It is mentioned by Azudin, Ismail and Taherali (2009) that knowledge sharing helps organizations achieve competitive advantage as they create new knowledge that can accelerate improvements in business process and technology. Furthermore, knowledge sharing enables active discussion and engagement among employees as they talk about know-what and know-how practices. As employees express their creative ideas, they are contributing and directing the organization towards growth and sustainable development. Ngah and Ibrahim (2010) further denoted that being the most important procedure in the KM process, knowledge sharing is directly correlated with organizational performance as it puts emphasis on the conversion of knowledge infrastructures including technology and culture into organizational capabilities. As an element of knowledge sharing, collaboration between and among employees helps increase productivity and efficiency of employee performance. This results in improved organizational efficiency and performance.

\subsection{What is the relationship between knowledge sharing and organizational leadership?}

Leadership is deemed an important aspect in knowledge sharing, specifically because the style of the leader influences behaviour of employees. In particular, transformational leaders are deemed to have a clear positive impact on the knowledge sharing behaviour of employees as they lead and facilitate the process. According to Bradshaw, Chebbi and Oztel (2015), good leaders know and understand the importance of knowledge sharing in the organization which is why they highly encourage employees to be more open and to communicate to exchange knowledge. For one, transformational leaders are good at empowering employees through effective facilitation and motivation to contribute to the knowledge sharing process. Needless to say, it is contended that any type of leadership can lead to positive outcomes for knowledge sharing (please see the figure found on the next page) as long as the leader supports knowledge sharing and implements practices that improve employees' perception and behaviour regarding the process. As organizations have become more knowledge-based nowadays, leaders have already recognized how knowledge sharing behaviour can improve efficiency in knowledge management which in turn, positively impacts organizational performance. As indicated by Mishra and Pandey (2018), leaders take on several roles and it is very important that they help improve knowledge sharing in the organization through openness and communication as this will be a factor leading to employee and organizational growth. 


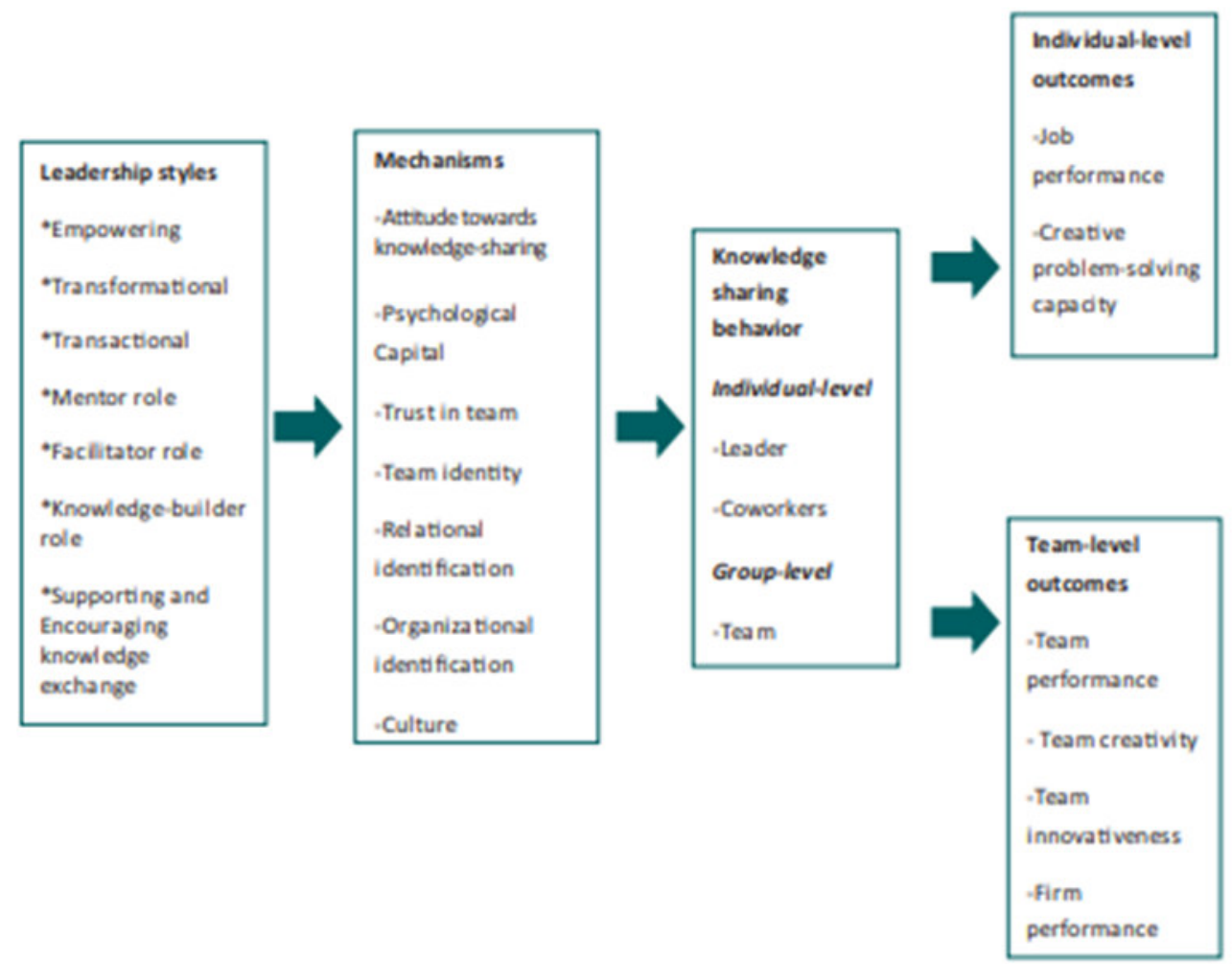

Figure 3: Theoretical framework for leadership and knowledge sharing research Source: Mishra \& Pandey, 2018

\subsection{What are the issues about knowledge sharing? What are their respective solutions?}

There is also a fair share of dilemmas encountered in knowledge sharing which can hinder the potential benefits it yields. For one, there are public good and social dilemmas that every organization needs to take into consideration. Public good dilemma is an issue related to how the knowledge shared can equally benefit the organization and the individual while social dilemma is concerned with the possibility of "free-ride". This means that a person can just wait for the others to contribute because he/she will be reaping the same rewards and benefits, even without doing anything. Other problems include high level of internal competition and limited know-how on the use of technology (Koulikov, 2011). All these can create an adverse effect on the practice of knowledge sharing in the organization, thereby driving negative behaviours such as resistance to the process and decline of collaboration and engagement from employees.

Nonetheless, there are possible solutions to these challenges. As Koulikov (2011) mentioned, organizations need to implement and maintain a system wherein employees can share, exchange and access knowledge-based resources. The system must also promote communication, openness and collaboration between and among the employees, emphasizing that knowledge sharing is more effective with these three attributes in play. Furthermore, strengthening the organizational culture is a key element in the success of knowledge sharing and in knowledge management as a whole. This culture must embody significant values such as ethics and integrity to make sure that there is fairness on how the employees are rewarded, that is, individual contributions must be recognized to avoid the aforementioned dilemmas. Also, integrating ethics into the organizational culture can motivate employees and create better relationships in the workplace as they are more collaborative and more involved in the knowledge sharing process.

\section{Discussion \& Conclusion}

This research emphasizes the role of knowledge sharing in knowledge management process and its impacts in the organization. Through the use qualitative research, we analyze information from existing studies to determine the significance of knowledge sharing in improving organizational performance and efficiency. The main highlights in this research are as follows: (1) KS is the most important part in the KM process and is a driving factor to improvement of KM environment and efficiency; (2) KS helps organizations improve their performance, as employees generate ideas that can propel innovations, organizational growth and sustainability; (3) KS can be promoted through the right leadership style and approach - one that is supportive and encouraging of knowledge sharing behaviours among employees; and (4) the issues surrounding KS such as tendency of free 
ride, limited technological know-how and intense internal competition can be addressed through an effective KS system and improved organizational culture. The information presented in this study can be explored in future researches, particularly the one pertaining to the relationship between knowledge sharing and leadership. Nonetheless, this study has failed to include the correlation between knowledge sharing, employee motivation and performance, as this topic is also reflective of the extended role of knowledge sharing in the efficiency, satisfaction and retention of employees.

\section{References}

Alqaryouti, O., Alqudah, A., \& Shaalan, K. (2016). Effective Knowledge Management in Projects: Characteristics and Integration. In Information and Knowledge Management (Vol. 6, No. 8, pp. 27-33).

Azudin, N., Ismail, M.N. \& Taherali, Z. (2009). Knowledge sharing among workers: a study on their contribution through informal communication in Cyberjaya, Malaysia. Knowledge Management \& ELearning: An International Journal 1(2), pp. 139-162.

Barriers - Three Blurry Terms in KM. The Electronic Journal of Knowledge Management 10(1), pp. 81-91.

Bradshaw, R., Chebbi, M. \& Oztel, H. (2015). Leadership and Knowledge Sharing. Asian Journal of Business Research, pp. 1-20.

Fteimi, N. \& Lehner, F. (2016). Main Research Topics in Knowledge Management: A Content Analysis of ECKM Publications. The Electronic Journal of Knowledge Management 14(1), pp. 5-17.

Gonzalez, K.V.D. \& Martns, M.F. (2014). Knowledge Management: an Analysis From the Organizational Development. Journal of Technology Management \& Innovation 9(1), pp. 131-147.

Greenhoot, A.F. \& Dowsett, C. (2012). Secondary Data Analysis: An Important Tool for Addressing Developmental Questions. Journal of Cognition and Development 13(1), pp. 2-18.

Intezari, A., Taskin, N. \& Pauleen, D. (2017). Looking beyond knowledge sharing: an integrative approach to knowledge management culture. Journal of Knowledge Management 21(2), pp. 492-515.

Koulikov, M. (2011). Emerging Problems in Knowledge Sharing and the Three New Ethics of Knowledge Transfer. Knowledge Management \& E-Learning: An International Journal 3(2), pp. 237-250.

Kumar, A.A. \& Kalva, U.K. (2015). KNOWLEDGE MANAGEMENT : A REVIEW. International Journal of Academic Research in Social Sciences \& Humanities (IJARSH) 1(1), pp. 9-17.

Lee, J. (2018). The Effects of Knowledge Sharing on Individual Creativity in Higher Education Institutions: Socio-Technical View. Administrative Sciences 8(21), pp. 1-16.

Mishra, M. \& Pandey, A. (2018). The impact of leadership styles on knowledge-sharing behavior: a review of literature. Development and Learning in Organizations: An International Journal, n.p.

Mohajan, H.K., Islam, S. \& Shome, F. (2017). Knowledge Sharing Enhances Knowledge Management Environment and Efficiency. Journal of Environmental Treatment Techniques 5(2), pp. 78-82.

Ngah, Rohana and Ibrahim, Abdul Razak (2010) The effect of knowledge sharing on organizational performance in small and medium enterprises. In: Knowledge Management International Conference 2010 (KMICe2010), 25-27 May 2010, Kuala Terengganu, Malaysia.

Pangil, F. \& Nasurddin, A. (2013). Knowledge and the importance of knowledge sharing in organizations. In: Conference on Business Management Research 2013, December 11, 2013, EDC, Universiti Utara Malaysia, Sintok

Paulin, D. \& Suneson, K. (2012). Knowledge Transfer, Knowledge Sharing and Knowledge Barriers - Three Blurry Terms in KM. The Electronic Journal of Knowledge Management 10(1), pp. 81-91.

Rusuli, M.S.C. \& Tasmin, R. (2010). Knowledge Sharing Practice In Organization. In: International Conference on Ethics and Professionalism (ICEP 2010), 1-2 December 2010, Equatorial Hotel Bangi, Putrajaya.

Shakouri, N. (2014). Qualitative Research: Incredulity toward Metanarrativeness. Journal of Education and Human Development 3(2), pp. 671-680.

Turner, J.R., Zimmerman, T. \& Allen, T. (2012). Teams as a Process for Knowledge Management. Journal of Knowledge Management 16(6), pp. 1-31.

Zheng, T. (2017). A Literature Review on Knowledge Sharing. Open Journal of Social Sciences 5, pp. 51-58. 